\title{
Three-Dimensional Needle Steering Using Automated Breast Volume Scanner (ABVS)
}

\author{
Momen Abayazid ${ }^{*+, \S, \pi}$, Pedro Moreira*, Navid Shahriari*, Anastasios Zompas*, \\ Sarthak Misra ${ }^{*,+\neq}$ \\ ${ }^{*}$ Department of Biomechanical Engineering, University of Twente, P. O. Box 217, 7500 AE, Enschede \\ The Netherlands \\ ${ }^{\dagger}$ Center for Medical Imaging - North East Netherlands \\ University of Groningen, University Medical Center Groningen \\ Hanzeplein 1, 9713 GB Groningen, The Netherlands \\ ${ }^{\ddagger}$ Department of Biomedical Engineering, University of Groningen and University Medical Center Groningen \\ Antonius Deusinglaan 1, 9713 AV, Groningen, The Netherlands
}

\begin{abstract}
Robot-assisted and ultrasound-guided needle insertion systems assist in achieving high targeting accuracy for different applications. In this paper, we introduce the use of Automated Breast Volume Scanner (ABVS) for scanning different soft tissue phantoms. The ABVS is a commercial ultrasound transducer used for clinical breast scanning. A preoperative scan is performed for three-dimensional (3D) target localization and shape reconstruction. The ultrasound transducer is also adapted to be used for tracking the needle tip during steering toward the localized targets. The system uses the tracked needle tip position as a feedback to the needle control algorithm. The bevel-tipped flexible needle is steered under ABVS guidance toward a target while avoiding an obstacle embedded in soft tissue phantom. We present experimental results for 3D reconstruction of different convex and non-convex objects with different sizes. Mean Absolute Distance (MAD) and Dice's coefficient methods are used to evaluate the 3D shape reconstruction algorithm. The results show that the mean MAD values are $0.30 \pm 0.13 \mathrm{~mm}$ and $0.34 \pm 0.17 \mathrm{~mm}$ for convex and non-convex shapes, respectively, while mean Dice values are $0.87 \pm 0.06$ (convex) and $0.85 \pm 0.06$ (non-convex). Three experimental cases are performed to validate the steering system. Mean targeting errors of $0.54 \pm 0.24,1.50 \pm 0.82$ and $1.82 \pm 0.40 \mathrm{~mm}$ are obtained for steering in gelatin phantom, biological tissue and a human breast phantom, respectively. The achieved targeting errors suggest that our approach is sufficient for targeting lesions of $3 \mathrm{~mm}$ radius that can be detected using clinical ultrasound imaging systems.
\end{abstract}

Keywords: Ultrasound; reconstruction; image processing.

\section{Introduction}

Needle insertion into soft tissue is a minimally invasive procedure used for diagnostic and therapeutic purposes

Received 26 June 2015; Revised 3 December 2015; Accepted 29 January 2016; Published 31 March 2016. Published in JMRR Special Issue on Image-Guided Intelligent Interventions. Guest Editors: Jayender Jagadeesan and Junichi Tokuda.

Email Address: \$mabayazid@bwh.harvard.edu

" Present address: Brigham and Women's Hospital, Harvard Medical School, 75 Francis Street, ASB1-L1-Room 050, Boston, MA 02115, USA. NOTICE: Prior to using any material contained in this paper, the users are advised to consult with the individual paper author(s) regarding the material contained in this paper, including but not limited to, their specific design(s) and recommendation(s). such as biopsy and brachytherapy, respectively. Examples of diagnostic needle insertion procedures are liver and lung biopsies to detect tumors [1, 2]. Therapeutic applications of needle insertion include brachytherapy of cervical, prostate and breast cancers [3]. Inaccurate placement may result in misdiagnosis and unsuccessful treatment during biopsy and brachytherapy, respectively. Errors in needle placement can be caused by inaccurate target localization. Initial target localization is of utmost importance for accurate insertions. Imaging modalities such as ultrasound, magnetic resonance (MR) and computed tomography (CT) are often used during needle insertion procedures to determine the positions of the needle and the target [4]. X-ray based imaging modalities 
such as fluoroscopy, CT and mammography are used to localize lesions [5]. Such imaging techniques expose the patient to undesirable doses of ionizing radiation [6]. MR imaging suffers from low refresh rate and incompatibility with magnetic materials and tools [7]. The spatial resolution of three-dimensional (3D) ultrasound images is limited [8] with respect to CT and MR, therefore twodimensional (2D) ultrasound is commonly used since it is real time, inexpensive and does not expose the patient to harmful radiations. Needle-guides are usually attached to the ultrasound transducer to facilitate needle visualization, and hence control during insertion applications such as biopsy [9]. Robotic assistance for needle-guidance provides improved lesion localization and targeting accuracy [10].

\subsection{Robotic control of ultrasound transducers}

Several researchers designed robotic ultrasound systems for lesion and needle detection insertion procedures [11-13]. An example of an early study that explores the advantage of robotic ultrasound systems is the development of Hippocrate, a robotic arm for medical applications with force feedback [14]. One of the applications of Hippocrate is the manipulation of an ultrasound transducer on a patient's skin to automatically reconstruct the 3D profile of arteries. Nadeau and Krupa developed a visual servoing method to control an industrial robotic system equipped with an ultrasound transducer [15]. Janvier et al. presented an ultrasound system to 3D reconstruct the shape of in vitro stenoses using an industrial robotic arm with force feedback [16]. Chatelain et al. developed a real-time needle tracking method by servoing images obtained from a 3D ultrasound transducer [17]. Vrooijink et al. introduced a three-degrees-of-freedom Cartesian robot for controlling the ultrasound transducer for scanning flat surfaces and 3D needle tip tracking [12]. Abayazid et al. designed a rotational mechanism using force/torque feedback that controls a 2D transducer to scan curved surfaces and developed an algorithm to localize the target and reconstruct its 3D shape [18]. The reconstruction was done by computing the convex hull of the point cloud obtained with the contour points of each planar cross section. However, this algorithm cannot reconstruct the shapes of non-convex tumors or anatomical obstacles. In the previously mentioned studies, robotic devices were used to control the ultrasound transducer during the scanning process. It is a challenging task to bring these mechanical and industrial robotic systems to the clinical environment due to safety restrictions. In the current study, we propose a system that can replace such robotic devices by a commercial ultrasound transducer which is the Automated Breast Volume Scanner (ABVS) (Fig. 1). This transducer is currently used in clinical settings, and it

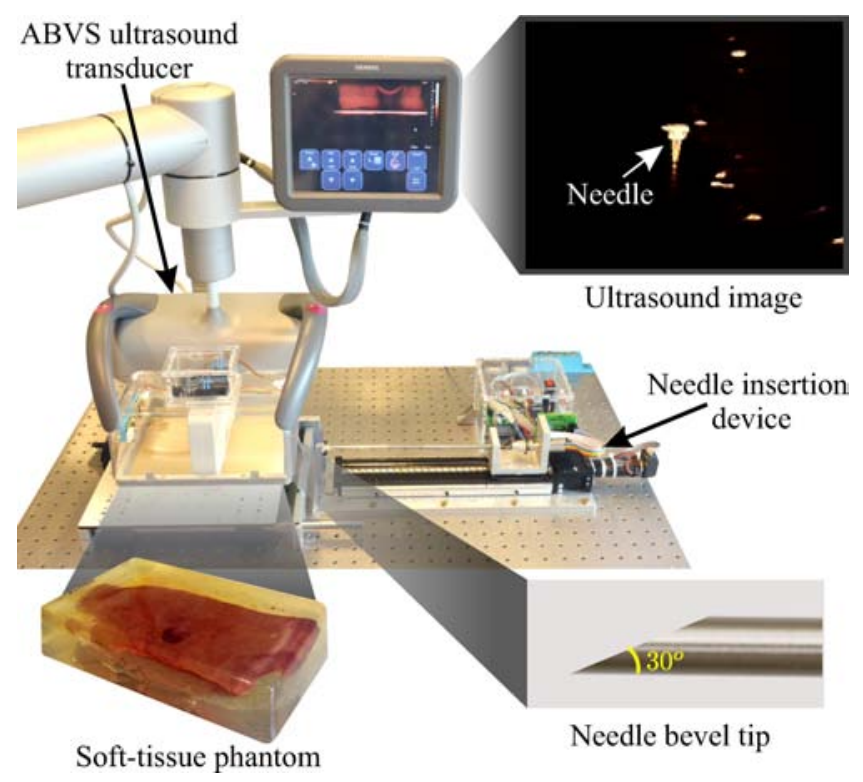

Fig. 1. The experimental setup shows the needle insertion device ND and the ABVS. The ultrasound image is used for three-dimensional needle tracking. The needle is inserted into a soft tissue phantom including biological tissue. The ABVS is used for preoperative scanning of the phantom and intraoperative bevel-tipped needle tip tracking. The associated custom-built electronics is used to synchronize the ABVS with the reconstruction and steering systems.

performs an automated scan of the human breast to generate a 3D volume of the scanned region. Furthermore, the proposed system adapts the commercial ultrasound system for needle tracking during the insertion procedure. More importantly, our system can be used not only for breast biopsy but also adapted for other various types of needle-based procedures.

\subsection{Flexible needle steering}

Flexible needles are introduced as they improve the steerablity and allow maneuvering around sensitive and hard tissue such as blood vessels and bones, respectively [19-21]. Such needles commonly have bevel tips that naturally deflect during insertion into soft tissue due to the asymmetric forces acting on their tips [22]. The direction of needle deflection is controlled by rotating the needle about its insertion axis to steer it towards a certain target location. In previous studies, control algorithms were developed for needle steering in 2D and 3D space. DiMaio and Salcudean presented a path planning and control algorithm that related the needle motion at the base (outside the soft tissue phantom) to the tip motion inside the tissue in 2D space [23]. Vrooijink et al. introduced an ultrasound-guided needle steering system using duty-cycle technique where the needle is inserted at constant velocity [12]. The transducer scanning velocity was controlled to keep the needle tip visible in the 
ultrasound image. In the current study, we are using a commercial ultrasound transducer which scans with a constant velocity. Therefore, we apply a closed-loop control to adjust the insertion velocity in order to keep the needle tip always in the image-plane for steering and path planning. Several 3D path planning algorithms have been introduced based on Rapidly exploring Random Trees (RRTs) for obstacle avoidance [24, 25]. The algorithm developed by Patil and Alterovitz is integrated to compute feasible collision-free paths in 3D-space [25].

\subsection{Contributions}

In the current study, we develop a system (Fig. 1) that performs ultrasound scanning of various soft tissue phantoms to localize and reconstruct different target and obstacles shapes. The ABVS system then tracks a beveltipped flexible needle intraoperatively to steer it toward the localized target in 3D space while avoiding the obstacles. The algorithms are validated by conducting insertion experiments into a soft tissue phantom and biological tissue (chicken breast and sheep liver) while avoiding virtual and real obstacles. We also steer the needle in a breast phantom used to train clinicians for ultrasound biopsy procedures. This breast phantom has similar mechanical and ultrasound visual properties of human breast tissue, and it also contains amorphous lesions. The major contributions of this work include:

- Replacing mechanical and industrial robotic devices used for ultrasound transducer control with patientfriendly commercial device for target localization and shape reconstruction.

- Developing algorithms for preoperative target localization, and reconstruction of non-convex target geometries.

- Adapting a clinically approved ultrasound imaging system (ABVS) to be used to 3D track the needle intraoperatively for various needle-based clinical procedures.

- Experimental evaluation of needle steering towards a physical target while avoiding an obstacle in different soft tissue phantoms.

\section{Target Localization and Shape Reconstruction}

In this section, we present the algorithms developed for 3D target localization and shape reconstruction. Evaluation methods are also described to validate the proposed reconstruction algorithm. The ABVS system is used to scan soft tissue phantoms containing targets with various 3D geometries and sizes.

The soft tissue phantom is preoperatively scanned using a Siemens Acuson S2000 (Siemens AG, Erlangen, Germany) ultrasound device to localize the target and reconstruct its shape. Ultrasound images are obtained using the ABVS system (Siemens Medical Solutions, Mountain View, CA,
USA). The ABVS scanner is composed of a cage that contains a transducer (14LBV). This transducer translates automatically using a linear stage with constant velocities of $1.55 \mathrm{~mm} / \mathrm{s}$ or $2.55 \mathrm{~mm} / \mathrm{s}$. The transducer has 768 elements and a frequency bandwidth of 5-14 MHz. The width of the transducer is $154 \mathrm{~mm}$ and its maximum display depth is $60 \mathrm{~mm}$. The frame rate of the system is 25 frames per second, and its voxel size is $0.09 \times 0.16 \times 0.44 \mathrm{~mm}^{3}$ along the axial, lateral and elevation planes, respectively.

\subsection{Registration and synchronization}

The ultrasound transducer is initially located in the middle position of the ABVS cage then it moves to one ends of the cage. The scan starts as soon as the transducer hits the end of the cage, and the scan is completed as the transducer hits the other end. The ABVS system is adapted by fixing internally two buttons on each end of the cage. These buttons are connected to a microcontroller board (Arduino Uno, Arduino, Italy) to determine the start time of the scan. The ultrasound transducer presses the buttons when it reaches one end of the cage. As soon as the button is released, which means that the transducer started moving from one end to the other, the algorithm starts capturing the images and assigns them to their corresponding transducer positions. The images are captured every $40 \mathrm{~ms}$. The transducer position of each captured image frame is determined using the starting time and the scanning velocity. The output data is then processed for target localization and shape reconstruction.

\subsection{Localization}

The images and their locations are analyzed to determine the frames that include the target. First, each image is inverted, and then the contrast is enhanced using contrast-limited adaptive histogram equalization [26]. The images are then converted to a binary image by adaptive thresholding. Closed object selection is performed to remove speckles and other image artifacts, resulting in the segmented cross-sectional views of the target. The centroid of the target cross section of each image frame is calculated using image moments $[27,28]$. The centroid of the target volume is computed using all the centroids of the image frames that contain the target. The contour points of each segmented image is extracted. The target reconstruction is accomplished using the point cloud representing the contour coordinates of the segmented ultrasound images. The point cloud is then used to reconstruct the 3D target shape.

\subsection{Target reconstruction algorithm}

The algorithms presented in our previous study could only reconstruct convex 3D target shapes [18]. In the 
current study, we developed an algorithm that can reconstruct different non-convex and convex volumes. First, the points of each image are sorted to draw the contour of the 2D section by linking each point to the next one. The outer points of the convex hull are determined [29]. Points that are not part of the convex hull are used for minimizing the length of the contour when it is possible. The step of minimizing the contour length allows the algorithm to reconstruct non-convex shapes. This results in connected points in each image frame (cross section of the target) (Fig. 2(c)). The consecutive contours are then connected to produce triangular meshing elements. Each triangular meshing element consists of one segment on one contour and two segments linking this contour to the next one. The sorting of points in the previous step facilitates the meshing process. The heuristic method presented by Ganapathy and Dennehy is used to connect different contours and forming the meshing element [30]. The meshing elements are then added to the surface connecting the contours (Fig. 2(d)). In the last reconstruction step, the two planar cross sections at both ends of the volume are meshed to close the 3D shape. The target reconstruction algorithm is evaluated by quantifying the spatial agreement of different convex and nonconvex 3D shapes with the 3D drawing of the molds designed for making the shapes. Dice's coefficient and Mean Absolute Distance (MAD) methods are used for shape comparison (Fig. 2(e)) [31, 32]. The results of the evaluation methods are presented in Section 4. The location of the reconstructed target is used as an input to the control algorithm to steer the needle towards the target.

\section{3D Needle Steering}

The ultrasound-based needle tracking algorithm provides the path planning and needle control algorithms with the tip position during insertion. In this section, the 3D needle tip tracking and control algorithms are described.

\subsection{Needle tip tracking}

The ABVS ultrasound system scans the phantom with a constant velocity. The ultrasound images are used to visualize the cross-section of the needle tip during insertion. A proportional-integral-derivative (PID) controller is implemented to keep the needle tip in the ultrasound image frame by adjusting the needle insertion velocity. The needle tracking algorithm is divided into two main parts. First, the image processing part that localizes the needle tip in ultrasound images. Second, the needle velocity control that synchronizes the needle and transducer velocities.

\subsubsection{Image processing}

The ultrasound transducer is placed perpendicular to the needle insertion direction (Fig. 3). The resulting ultrasound image shows a radial cross-sectional view of the needle. An image processing algorithm is used to localize the needle in the ultrasound images. The image processing is divided to pre-processing and post-processing phases. In the pre-processing phase, the ultrasound images are enhanced and filtered to eliminate speckles using a sequence of segmentation techniques, including Gaussian filtering, dilation and thresholding. In the postprocessing phase, the contours of all objects in the image are determined. These objects include the needle cross section and other artifacts that appear mainly in biological tissue. Fourier descriptors are used to extract the needle contour features in order to distinguish the needle from other artifacts [33]. The contour features are extracted from a sample image prior to the experiments.

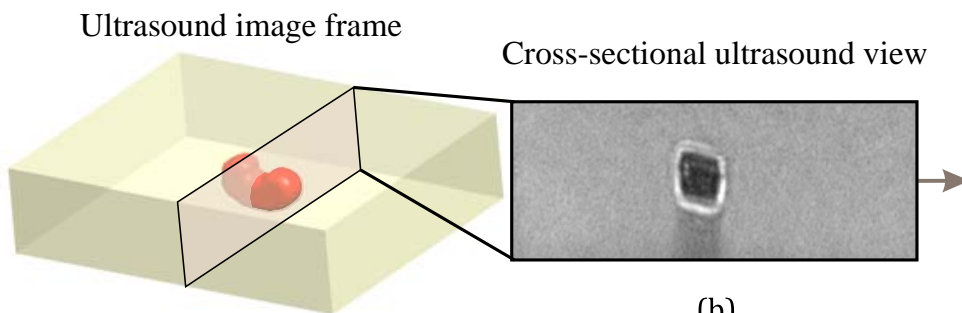

(a)

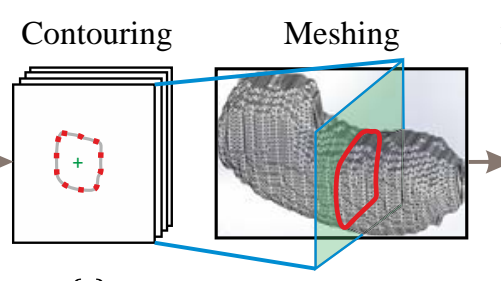

(c) (d)
3D shape comparison

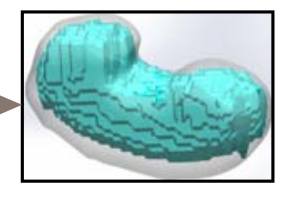

(e)

Fig. 2. (Color online) Target localization and shape reconstruction. (a) The soft tissue phantom is scanned and image frames that contain the target are segmented. (b) The target cross section appears darker than the surrounding tissue in the raw ultrasound images. (c) The contour points of the target cross section are obtained. The points are sorted and the centroid of each contour is computed (green "+"). The points of each frame are then connected to define the contour shape. The ultrasound images are stacked together for 3D shape reconstruction. (d) The contours are connected together to form the mesh elements. (e) The 3D reconstruction algorithm is evaluated by comparing the reconstructed shape (green) with reference 3D shape (greyish) using MAD and Dice's coefficient methods. 


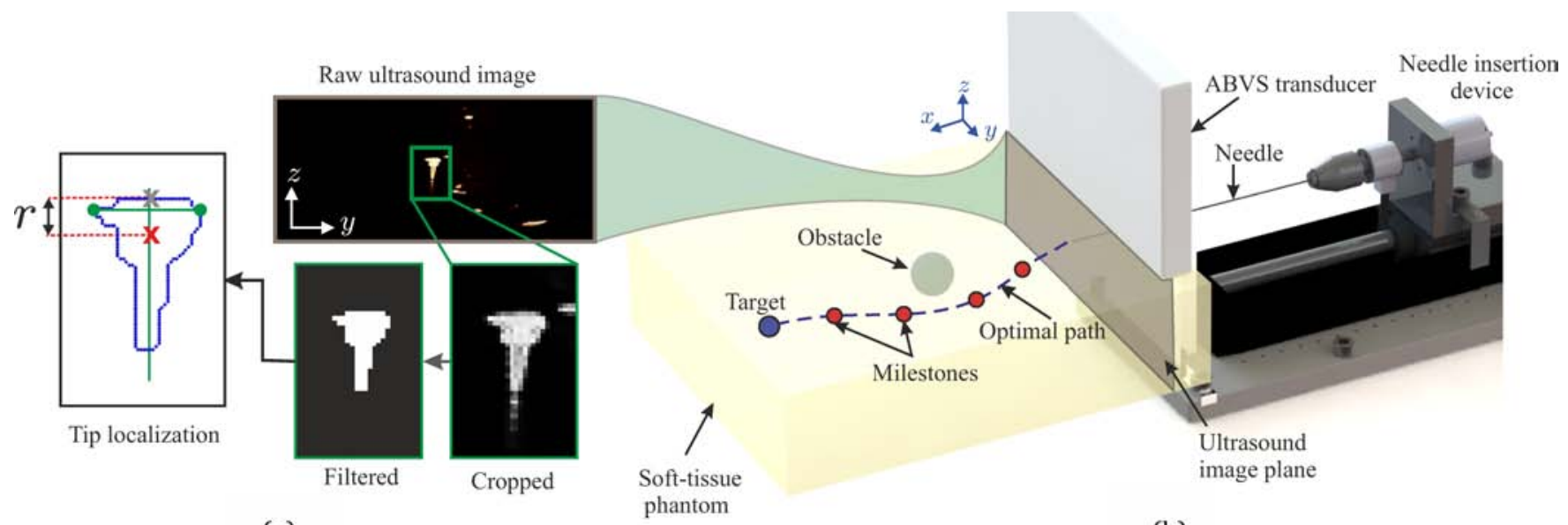

(a)

(b)

Fig. 3. (Color online) The needle tip pose is determined in 3D space using a 2D ABVS transducer positioned to visualize the needle tip where the ultrasound image plane is perpendicular to the needle insertion axis ( $x$-axis). (a) The region of interest that contains the needle tip is cropped and filtered and then contour of the needle cross section is obtained. The $y$-coordinate of the needle center is the midpoint between the maximum and minimum $y$-coordinate values (green circles) of the points along the contour. Since the needle radius is known, we subtract the needle radius ( $r$ ) from the maximum $z$-coordinate (gray " $\times$ ") along the contour to compute the center of the needle. The needle tip position (red " $\times$ ") is an input of the path planning algorithm to generate the optimal needle path to avoid the obstacle and reach the target. (b) The path planner generates milestones along the path, and the control algorithm steers the needle using the milestones to move along the planned trajectory.

The needle cross-section contour is then used to localize its tip as shown in Fig. 3(a).

\subsubsection{Velocity control}

The ABVS transducer performs breast scans with a constant velocity of $1.55 \mathrm{~mm} / \mathrm{s}$. Therefore, the $x$-component of the needle tip velocity $\left(v_{x_{\text {tip }}}\right)$ has to be controlled to keep the needle tip in the ultrasound image plane. Assuming that the needle is not compressed along its insertion axis, the $x$-component of the needle tip velocity $\left(v_{x_{\text {tip }}}\right)$ is calculated by $v_{x_{\text {tip }}}=\sqrt{v_{i}^{2}+v_{y_{\text {tip }}}^{2}+v_{z_{\text {tip }}}^{2}}$, where $v_{i}$, $v_{y_{\text {tip }}}$ and $v_{z_{\text {tip }}}$ are the insertion velocity and the corresponding needle $y$ - and $z$-components of the tip velocity, respectively. The tip velocities $v_{y_{\text {tip }}}$ and $v_{z_{\text {tip }}}$ are estimated by the image processing algorithm. A Kalman observer is used to filter the tip position information and to estimate the needle tip position and velocity when the tip is not visible in the ultrasound image frame. The system states in the Kalman observer are the Cartesian needle tip positions and velocities [12]. The gains of the observer are tuned as presented by Vrooijink et al. [34]. The Kalman observer is also used to estimate the needle tip pose if it is not visible in the ultrasound image due to the presence of bones between the transducer and the needle.

The needle insertion velocity $\left(v_{i}\right)$ is adjusted by a PID controller to maintain the $x$-component of the needle tip velocity $\left(v_{x_{\text {tip }}}\right)$ in order to keep it equal to a desired velocity $\left(v_{x_{\text {des }}}\right)$, as shown in Fig. 4 . The desired velocity is increased if the needle is out-of-plane

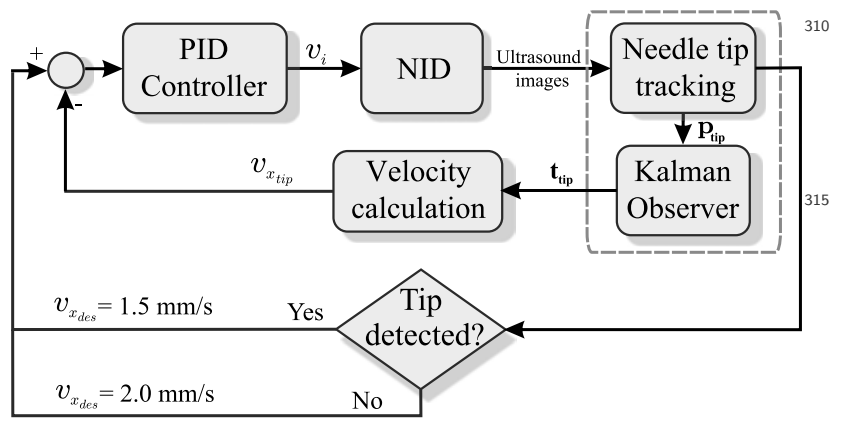

Fig. 4. A PID controller is implemented in order to keep the $x$ axis component of the needle tip velocity $\left(v_{x_{\text {tip }}}\right)$ equal to the desired velocity $\left(v_{x_{\text {des }}}\right)$. The needle tip position ( $\left.\mathbf{p}_{\text {tip }}\right)$ detected by the tracking algorithm is used as input for the Kalman observer to provide the output vector composed by both the needle tip positions ( $\left.\mathbf{p}_{\text {tip }}\right)$ and velocities $\left(\mathbf{t}_{\text {tip }}\right)$. The controller output is the insertion velocity $\left(v_{i}\right)$ performed by the NID. The velocity $\left(v_{x_{\text {des }}}\right)$ is increased if the needle is not detected in the ultrasound image plane, and it is decreased if the needle is detected in the image plane.

(i.e. the tip is not detected, and the needle is lagging) or decreased if the needle is in-plane (i.e. the tip is detected). This step is performed to ensure that the tracking algorithm detects the tip of the needle and not its shaft. The gains of the PID controller are experimentally tunned to assure fast reaction, prevent overshoot and guarantee accurate needle tip tracking. The needle pose obtained by the tracking algorithm is the main input to the control algorithm and the path planner. 


\subsection{D needle path planning and control}

The target and obstacle positions obtained from the ABVS preoperative scan (presented in Sec. 2), and the needle tip pose computed intraoperatively from the tracking algorithm (presented in Sec. 3) are used as input to the path planning and control algorithms.

The 3D path planning algorithm generates the optimal trajectory to steer the needle toward the target while avoiding an obstacle in 3D space [25]. The planning algorithm is based on the RRT approach, which is a sampling-based method for path planning [35]. The optimal trajectory is replanned every second during needle insertion to compensate for uncertainties or disturbances in the system or environment. The path planner outputs a sequence of milestones along the optimal path. These milestones are used as an input to the needle control algorithm. The algorithm steers the needle tip toward the first milestone. As soon as a milestone is reached, the control algorithm steers the needle toward the next milestone until the target is reached (Fig. 3(b)).

The needle is assumed to move along arcs during its insertion into a soft tissue phantom [22]. The direction of motion along the arc depends on the bevel tip orientation, which is adjusted by rotating the needle about its axis at the base. The needle tip pose obtained from the tracking algorithm, the milestone positions computed using the planner and the final target position calculated from the preoperative scan are inputs of the control algorithm. We refer the reader to the work by Patil and Alterovitz and Abayazid et al. for additional details on the planning and control algorithms $[25,36]$.

\section{Experiments}

In this section, we present the experimental setup used for scanning the soft tissue phantom and also for needle steering. Subsequently, the experiments performed for evaluation of the reconstruction and control algorithms are described.

\subsection{Setup}

The experimental setup is divided into two parts. First, the two-degrees-of-freedom insertion device which allows the needle to be inserted and rotated about its axis [37]. Second, the ABVS transducer that scans the soft tissue phantom to localize the target and reconstruct its shape, as shown in Fig. 1. The ABVS is also used for needle tip tracking during the steering procedure. The needle is inserted into a soft tissue phantom made up of a gelatin mixture, chicken breast and sheep liver. The height of the gelatin phantom is $5.5 \mathrm{~cm}$, and targets are placed $2-3.5 \mathrm{~cm}$ from the phantom's surface. We also steer the needle in a commercial human breast phantom (CIRS, Norfolk, USA). This breast phantom contains lesions of different shapes and sizes and it is used for ultrasound biopsy training. The flexible needle is made of Nitinol alloy (nickel and titanium). The Nitinol needle has a diameter of $0.5 \mathrm{~mm}$ with a tip bevel angle of $30^{\circ}$.

\subsection{Experimental plan}

Various experimental scenarios are conducted to validate the proposed target reconstruction and control algorithms.

\subsubsection{Shape reconstruction}

We assess the target reconstruction algorithm by scanning different 3D geometries. Targets are made of an aqueous solution of $20 \mathrm{wt} . \%$ polyvinyl alcohol (PVA) (Sigma-Aldrich Chemie B.V., The Netherlands) to mimic the ultrasound visual properties of breast lesions [38,39]. The solution is cast in 3D printed mold with cavities of bean shapes and spherical shapes of $3 \mathrm{~mm}$ and $4 \mathrm{~mm}$ radius. The final material is prepared undergoing two freeze and thaw cycles. The output of the target reconstruction algorithm is compared to the 3D drawings used for designing the 3D printed molds. MAD and Dice's coefficient methods are used for shape comparison. The reconstruction algorithm is applied on five samples of each target shape.

\subsubsection{Steering}

The phantom is scanned preoperatively to localize the obstacle and target in the soft tissue phantom. The needle is placed in a new insertion point of the phantom for each experimental trial. The target and obstacle positions vary depending on the results of the localization algorithm applied before every experimental trial. The control algorithm moves the needle along the generated path to avoid the obstacle and reach the target. Three experimental cases are performed to evaluate the needle steering system. Each sub-experimental case is performed five times.

- Case 1: The needle is steered toward a virtual target in (a) gelatin-based soft tissue phantom, (b) chicken breast and (c) sheep liver (Fig. 5(a)). This experimental case is performed to test the ability of the ABVS-guided control algorithm to steer the needle toward a certain position in different types of tissues.

- Case 2: The needle is steered towards a physical target while avoiding a physical obstacle embedded in (a) gelatin-based soft tissue phantom and (b) chicken breast (Fig. 5(b)). This experimental case is performed to validate the path planning and $3 \mathrm{D}$ localization algorithms. 


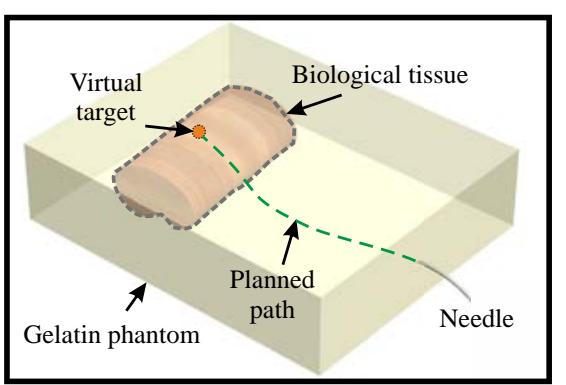

Case 1

(a)

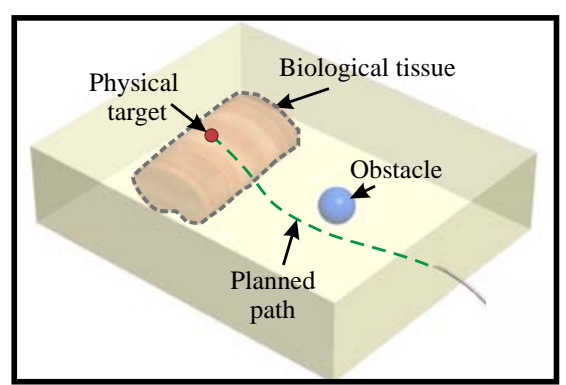

Case 2

(b)

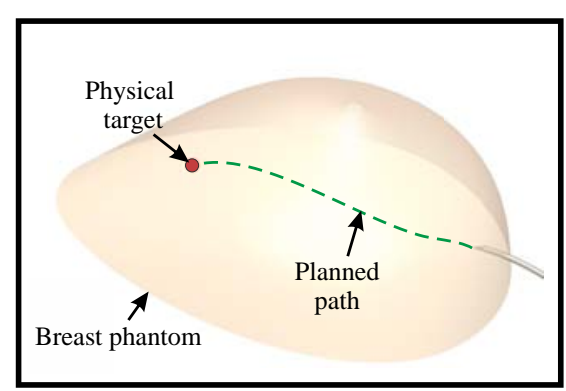

Case 3

(c)

Fig. 5. Experimental cases. (a) The needle is steered toward a virtual target in a gelatin-based soft tissue phantom (Case 1(a)), chicken breast (Case 1(b)) and sheep liver (Case 1(c)). (b) The needle is steered towards a physical target while avoiding a physical obstacle in gelatin-based soft tissue phantom (Case 2(a)) and chicken breast (Case 2(a)). (c) The needle is steered towards a physical target while avoiding a physical obstacle in a human breast tissue phantom (Case 3).

- Case 3: The needle is steered towards a physical target in a breast tissue phantom that consists of several lesions (Fig. 5(c)). The phantom is partially embedded in gelatin to restrict its motion during the scan. This experimental case is performed to test ability of the system to steer the needle in an environment similar to human breast biopsy.

\subsection{Results}

The results of the MAD and Dice's coefficient evaluation methods for reconstruction algorithm are presented in Table 1 . The results show that the MAD values are less than $0.5 \mathrm{~mm}$ for all 3D geometrical shapes while Dice's coefficient values are around 0.87 and 0.80 for convex and non-convex 3D shapes, respectively. These results show accurate shape reconstruction for such needle insertion applications (i.e. biopsy and brachytherapy).

In the steering experimental cases, the error is defined as the absolute distance between the tip and the center of the target that is localized preoperatively. The results of the experimental cases are presented in Table 2. The needle tip reaches the target in each

Table 1. The target reconstruction is evaluated using the MAD and Dice's coefficient geometric comparison methods for shapes with various sizes. The dimensions of the bean shape are represented by the length, width and thickness, respectively. The target reconstruction algorithm is applied on five samples of each shape.

\begin{tabular}{lccc}
\hline & Geometry & MAD $(\mathrm{mm})$ & Dice \\
\hline Sphere & $3 \mathrm{~mm}$ radius & $0.28 \pm 0.12$ & $0.87 \pm 0.05$ \\
& $4 \mathrm{~mm}$ radius & $0.31 \pm 0.16$ & $0.87 \pm 0.07$ \\
Bean shape & $9.6 \times 5.6 \times 4 \mathrm{~mm}^{3}$ & $0.27 \pm 0.05$ & $0.87 \pm 0.03$ \\
& $12 \times 7 \times 5 \mathrm{~mm}^{3}$ & $0.26 \pm 0.06$ & $0.88 \pm 0.03$ \\
& $18 \times 10.5 \times 5.5 \mathrm{~mm}^{3}$ & $0.55 \pm 0.12$ & $0.80 \pm 0.07$ \\
\hline
\end{tabular}

experimental trial (according to the images obtained during insertion). The maximum targeting error is $2.53 \mathrm{~mm}$, and it is noted in Case 1(c). On the other hand, the minimum targeting error is $0.42 \mathrm{~mm}$, and it is observed in Case 1(a). The results show that the targeting error increases while steering in biological tissue, especially sheep liver, due to its inhomogeneity. The inhomogeneity of the biological tissue causes deviation of the needle from its predicted path and thus increased targeting error. The targeting error increases also while steering in the human breast phantom (Case 3) (with respect to the other Case 1 and Case 2) as the curvature of the bevel-tipped needle is limited in such soft tissue.

\section{Discussion}

This study introduces a needle steering system that uses the ultrasound-based ABVS system for scanning different soft tissue phantoms including biological tissue and human breast phantoms. The system combines preoperative 3D target localization and shape reconstruction algorithms with intraoperative needle tip tracking, path planning and ultrasound-guided control algorithms to

Table 2. The results of the needle path planning and steering experiments. The mean and standard deviation of the targeting error $\left(e_{t}\right)$ of each case are presented. Each experimental case is performed five times.

\begin{tabular}{llll}
\hline \multicolumn{1}{c}{ Case } & \multicolumn{1}{c}{ Description } & $e_{t}(\mathrm{~mm})$ \\
\hline Case 1 & (a) & Gelatin-based phantom & $0.42 \pm 0.14$ \\
& (b) & Chicken breast & $0.96 \pm 0.39$ \\
& (c) & Sheep liver & $2.53 \pm 0.40$ \\
Case 2 & (a) & Gelatin-based phantom & $0.66 \pm 0.26$ \\
& (b) & Chicken breast & $1.01 \pm 0.27$ \\
Case 3 & & Human breast phantom & $1.82 \pm 0.40$ \\
\hline
\end{tabular}


steer a bevel tip needle towards a physical target while avoiding a physical obstacle. The reconstruction algorithm is capable of estimating different convex and non-convex 3D shapes of various sizes.

The reconstruction algorithm is evaluated using MAD and Dice's coefficient methods. The reconstruction evaluation methods are applied on spherical (convex) and bean-shaped (non-convex) geometries.

The proposed system is a step to bring advanced robotic systems to clinical environments. The system uses clinically approved system (ABVS) for 3D target shape reconstruction and needle tip tracking. The system is validated using various soft tissue phantoms and biological tissue. The obtained results are promising and indicate the feasibility of using such a system in clinical practice. However, further improvements are still needed as real tumors are more challenging to detect in the patient's body. Advanced image processing techniques should be used to localize tumors and reconstruct their 3D shapes. These techniques include background intensity level determination and statistical segmentation methods. Clinical data sets can be used to validate the proposed algorithms and achieve improved reconstruction results. One of the limitations that should be considered is the shadowing effect in the ultrasound images below bone tissue. This effect can distort the image if bones are located between the transducer and the needle tip or target. The image can also be affected by the distance between the target and transducer. The ABVS system can be combined with other needle tracking modalities for robust tip localization. Furthermore, advanced imaging techniques can be used if the target is below bone tissue. The Kalman observer can estimate the needle position if it is not visible in the image for a few seconds but for longer periods different tracking methods can be used. The proposed system can be employed in prostate, liver and kidney interventions using linear and transrectal transducers for ultrasound guidance where the needle should avoid the sensitive vessels.

\section{Conclusions and Future Work}

The 3D shape reconstruction evaluation results show that the mean MAD values are $0.30 \pm 0.13 \mathrm{~mm}$ and $0.34 \pm 0.17 \mathrm{~mm}$ for convex and non-convex shapes, respectively, while mean Dice values are $0.87 \pm 0.06$ and $0.85 \pm 0.06$. Three experimental cases are performed to validate the needle tracking, path planning and control algorithms. The needle is inserted in gelatin-based softtissue phantoms, biological tissue (i.e. chicken breast and sheep liver) and also human breast phantoms. The phantoms are scanned before every experimental trial to localize the targets and obstacles. The experimental results show that needle avoids the obstacle and reaches the target in each experimental trial and the mean targeting errors range between $0.42 \pm 0.14 \mathrm{~mm}$ and $2.53 \pm 0.40 \mathrm{~mm}$. It is observed that the needle curvature in the human breast phantom is limited with respect to gelatin while the needle deflection in biological tissue varies due to its inhomogeneity. The results also show that the proposed system that uses ABVS ultrasound scanner which is compatible with clinical environments can achieve high targeting accuracy as the target is reached and the obstacle is avoided in all experimental trials.

The needle behavior in different tissues should be investigated for improved planning of the insertion procedure. Models should also be developed to estimate the target motion and shape deformation intraoperatively. The steering system can be extended to detect the patient movements that occur during needle insertion such as respiration and fluid flow. Preoperative planning can be introduced to optimize the insertion location and angle in order to minimize the insertion distance and facilitate the steering process. Clinical transducers can be designed and adapted to the ABVS for other needle insertion procedures. This opens the field for having automated scanners to assist in achieving improved targeting accuracy.

\section{Acknowledgments}

The authors would like to thank Mr. Marius Gras and Ms. Teresa Araújo for their assistance in performing the experiments. The authors would also like to thank Dr. Sachin Patil and Prof. Ron Alterovitz for providing the path planning algorithm, which was developed at the University of North Carolina at Chapel Hill, USA. This work was supported by funds from the Netherlands Organization for Scientific Research (NWO) Innovative Medical Devices Initiative (IMDI) - Project: USE (Ultrasound Enhancement) and NWO - Project number: 11204.

\section{References}

1. E. M. Boctor, M. A. Choti, E. C. Burdette and R. J. Webster III, Threedimensional ultrasound-guided robotic needle placement: An experimental evaluation, Int. J. Med. Robot. Comput. Assist. Surg. 4(2) (2008) 180-191.

2. L. B. Kratchman, M. M. Rahman, J. R. Saunders, P. J. Swaney and R. J. Webster III, Toward robotic needle steering in lung biopsy: A tendon-actuated approach, in Proc. Society of Photographic Instrumentation Engineers (SPIE), Medical Imaging: Visualization, Image-Guided Procedures, and Modeling, Vol. 7964 eds. K. H. Wong and D. R. Holmes III, Florida, USA, February 2011, pp. $79641 \mathrm{I}$ $(1-8)$.

3. P. Beddy, R. D. Rangarajan and E. Sala, Role of mri in intracavitary brachytherapy for cervical cancer: What the radiologist needs to know, Am. J. Roentgeno. 196(3) (2011) W341-W347.

4. R. Seifabadi, S.-E. Song, A. Krieger, N. Cho, J. Tokuda, G. Fichtinger and I. Iordachita, Robotic system for mri-guided prostate 
biopsy: Feasibility of teleoperated needle insertion and ex vivo phantom study, Int. J. Comput. Assist. Radiol. Surg. 7(2) (2012) 181-190.

5. D. Glozman and M. Shoham, Image-guided robotic flexible needle steering, IEEE Trans. Robot. 23(3) (2007) 459-467.

6. H. L. Fred, Drawbacks and limitations of computed tomography, Texas Heart Inst. J. 31(2) (2004) 345-348.

7. S. P. DiMaio, E. Samset, G. Fischer, I. Iordachita, G. Fichtinger, F. Jolesz and C. M. Tempany, Dynamic mri scan plane control for passive tracking of instruments and devices, in Medical Image Computing and Computer-Assisted Intervention, eds. N. Ayache, S. Ourselin and A. Maeder (Springer Berlin/Heidelberg, 2007), pp. 50-58.

8. P. M. Novotny, J. A. Stoll, N. V. Vasilyev, P. J. del Nido, P. E. Dupont, T. E. Zickler and R. D. Howe, Gpu based real-time instrument tracking with three-dimensional ultrasound, Med. Image Anal. 11 (5) (2007) 458-464.

9. C. Kim, D. Chang, D. Petrisor, G. Chirikjian, M. Han and D. Stoianovici, Ultrasound probe and needle-guide calibration for robotic ultrasound scanning and needle targeting, IEEE Trans. Biomed. Eng. 60(6) (2013) 1728-1734.

10. K. B. Reed, A. Majewicz, V. Kallem, R. Alterovitz, K. Goldberg, N. J. Cowan and A. M. Okamura, Robot-assisted needle steering, IEEE Robot. Autom. Mag. 18(4) (2011) 35-46.

11. A. Krupa, A new duty-cycling approach for $3 \mathrm{~d}$ needle steering allowing the use of the classical visual servoing framework for targeting tasks, in Proc. IEEE RAS EMBS Int. Conf. Biomedical Robotics and Biomechatronics, Sao Paulo, Brazil, August 2014, pp. 301-307.

12. G. J. Vrooijink, M. Abayazid, S. Patil, R. Alterovitz and S. Misra, Needle path planning and steering in a three-dimensional nonstatic environment using two-dimensional ultrasound images, Int. J. Robot. Research 33(10) (2014) 1361-1374.

13. Z. Neubach and M. Shoham, Ultrasound-guided robot for flexible needle steering, IEEE Trans. Biomed. Eng. 57(4) (2010) 799-805.

14. F. Pierrot, E. Dombre, E. Dégoulange, L. Urbain, P. Caron, S. Boudet, J. Gariépy and J. L. Mégnien, Hippocrate: A safe robot arm for medical applications with force feedback, Med. Image Anal. 3(3) (1999) 285-300.

15. C. Nadeau and A. Krupa, Intensity-based ultrasound visual servoing: Modeling and validation with 2-D and 3-D probes, IEEE Trans. Robot. 29(4) (2013) 1003-1015.

16. M.-A. Janvier, L.-G. Durand, M.-H. R. Cardinal, I. Renaud, B. Chayer, P. Bigras, J. de Guise, G. Soulez and G. Cloutier, Performance evaluation of a medical robotic 3D-ultrasound imaging system, Med. image Anal. 12(3) (2008) 275-290.

17. P. Chatelain, A. Krupa and M. Marchal, Real-time needle detection and tracking using a visually servoed $3 \mathrm{~d}$ ultrasound probe, Proc. IEEE Int. Conf. Robotics and Automation (ICRA), Karlsruhe, Germany, May 2013, pp. 1676-1681.

18. M. Abayazid, P. Moreira, N. Shahriari, S. Patil, R. Alterovitz and S. Misra, Ultrasound-guided three-dimensional needle steering in biological tissue with curved surfaces, Med. Eng. Phys. 37(1) (2015) 145-150.

19. A. Grant and J. Neuberger, Guidelines on the use of liver biopsy in clinical practice, J. Gastroente. Hepatol. 45(Suppl. IV) (1999) IV1IV11.

20. V. Kallem and N. J. Cowan, Image-guided control of flexible beveltip needles, in Proc. IEEE Int. Conf. Robotics and Automation (ICRA), Rome, Italy, April 2007, pp. 3015-3020.

21. N. J. Cowan, K. Goldberg, G. S. Chirikjian, G. Fichtinger, K. B. Reed, V. Kallem, W. Park, S. Misra and A. M. Okamura, Robotic needle steering: Design, modeling, planning, and image guidance, in Surgical Robotics (Springer US, 2011), pp. 557-582.
22. R. J. Webster III, J. S. Kim, N. J. Cowan, G. S. Chirikjian and A. M. Okamura, Nonholonomic modeling of needle steering, Int. J. Robot. Res. 25(5-6) (2006) 509-525.

23. S. P. DiMaio and S. E. Salcudean, Needle steering and model-based trajectory planning, in Proc. Int. Conf. Medical Image Computing and Computer-Assisted Intervention (MICCAI), Montral, Canada, Vol. 2878, November 2003, pp. 33-40.

24. J. Xu, V. Duindam, R. Alterovitz and K. Goldberg, Motion planning for steerable needles in $3 \mathrm{~d}$ environments with obstacles using rapidly-exploring random trees and backchaining, in Proc. IEEE Int. Conf. Automation Science and Engineering (CASE), Washington, DC, USA, August 2008, pp. 41-46.

25. S. Patil and R. Alterovitz, Interactive motion planning for steerable needles in $3 \mathrm{~d}$ environments with obstacles, in Proc. IEEE RAS and EMBS Int. Conf. Biomedical Robotics and Biomechatronics (BioRob), Tokyo, Japan, September 2010, pp. 893-899.

26. E. D. Pisano, S. Zong, B. M. Hemminger, M. DeLuca, R. E. Johnston, K. Muller, M. P. Braeuning and S. M. Pizer, Contrast limited adaptive histogram equalization image processing to improve the detection of simulated spiculations in dense mammograms, J. Digit. Imaging 11(4) (1998) 193-200.

27. M.-K. Hu, Visual pattern recognition by moment invariants, IRE Trans. Inf. Theor. 8(2) (1962) 179-187.

28. M. R. Teague, Image analysis via the general theory of moments, J. Opt. Soc. Am. 70(8) (1980) 920-930.

29. F. P. Preparata and S. J. Hong, Convex hulls of finite sets of points in two and three dimensions, Commun. Assoc. Comput. Mach. 20(2) (1977) 87-93.

30. S. Ganapathy and T. G. Dennehy, A new general triangulation method for planar contours, SIGGRAPH Comput. Graph. 16(3) (1982) 69-75.

31. L. R. Dice, Measures of the amount of ecologic association between species, Ecology 26(3) (1945) 297-302.

32. S. Ghose, A. Oliver, R. Mart, X. Llad, J. Freixenet, J. Mitra, J. C. Vilanova and F. Meriaudeau, A hybrid framework of multiple active appearance models and gloal registration for 3d prostate segmentation in mri, in Proc. Society of Photographic Instrumentation Engineers (SPIE), Medical Imaging: Image Processing, Vol. 8314, eds. D. R. Haynor and S. Ourselin San Diego, CA, USA, February 2012, pp. 83140S (1-9).

33. C. T. Zahn and R. Z. Roskies, Fourier descriptors for plane closed curves, IEEE Trans. Comput. C-21(3) (1972) 269-281.

34. G. J. Vrooijink, M. Abayazid and S. Misra, Real-time three-dimensional flexible needle tracking using two-dimensional ultrasound, in Proc. IEEE Int. Conf. Robotics and Automation (ICRA), Karlsruhe, Germany, May 2013, pp. 1680-1685.

35. S. M. LaValle, Planning Algorithms (Cambridge University Press, 2006).

36. M. Abayazid, G. J. Vrooijink, S. Patil, R. Alterovitz and S. Misra, Experimental evaluation of ultrasound-guided 3d needle steering in biological tissue, Int. J. Comput. Assist. Radiol. Surg. 9(6) (2014) 931-939.

37. M. Abayazid, R. J. Roesthuis, R. Reilink and S. Misra, Integrating deflection models and image feedback for real-time flexible needle steering, IEEE Trans. Robot. 29(2) (2013) 542-553.

38. K. J. M. Surry, H. J. B. Austin, A. Fenster and T. M. Peters, Poly(vinyl alcohol) cryogel phantoms for use in ultrasound and MR imaging, Phys. Med. Biol. 49(24) (2004) 5529-5546.

39. W. Xia, D. Piras, M. Heijblom, W. Steenbergen, T. G. van Leeuwen and S. Manohar, Poly(vinyl alcohol) gels as photoacoustic breast phantoms revisited, J. Biomed. Opt. 16(7) (2011) 075002 (1-9). 